\title{
INTERPRETIVE SUMMARIES, OCTOBER 2010
}

Invited review: Lactobacillus helveticus - A thermophilic dairy starter related to gut bacteria. By Slattery et al., page 4435. Lactobacillus helveticus is a widely used species in the dairy industry and has been used for decades. We published the sequence of Lb. helveticus DPC4571 last year, which showed that this strain has a remarkable similarity to some intestinal lactobacilli. The existing body of literature shows how $L b$. helveticus improves dairy foods; for example, through its proteolytic systems. It is also used for the manufacture of dairy products containing bioactive peptides, such as those with anti-angiotensin converting enzyme activity.

Invited review: Modeling within-herd transmission of Mycobacterium avium subspecies paratuberculosis in dairy cattle: A review. By Marcé et al., page 4455. Models are a simplified representation of reality, and they have been used to study control strategies for paratuberculosis in dairy herds. To do this meaningfully, a model needs to be an acceptable representation of current knowledge on herd management and paratuberculosis. This review identifies those aspects in existing models that could be improved. These include (1) assessing the importance of contact structure within herds, (2) explicitly integrating calf-tocalf transmission, and (3) modeling indirect transmission via the environment instead of through contacts between animals, because Mycobacterium avium ssp. paratuberculosis can survive for extended periods outside the host.

Sensory and microbiological quality of yogurt drinks with prebiotics and probiotics. By Allgeyer et al., page 4471. As the popularity of yogurt products continues to grow, manufacturers are continually investigating value-added ingredients such as prebiotics and probiotics and their effect in yogurt in order to entice health-conscious consumers. The sensory changes of yogurt drinks with various types and amounts of prebiotics and probiotics were evaluated by a trained panel. Survival of the probiotics in the drink during $30 \mathrm{~d}$ of refrigerated storage declined. The results of this study can provide quality information for yogurt manufacturers wanting to incorporate these healthful ingredients into their dairy products.

Effect of bovine lactoferrin addition to milk in yogurt manufacturing. By Franco et al., page 4480. Lactoferrin is an iron-binding protein found mainly in milk. It exhibits several biological activities, some of them associated with iron absorption and host defense. For this reason, lactoferrin is used as a supplement in some functional food products. The objective of this work was to study the effect of milk supplementation with lactoferrin of different iron saturations and at different concentrations on the manufacturing and characteristics of yogurt. Our results allow us to conclude that supplementation with lactoferrin did not substantially affect the physicochemical characteristics and content of living lactic bacteria of yogurt and that yogurt is a good vehicle for lactoferrin.

Potent antilisterial cell-free supernatants produced by complex red-smear cheese microbial consortia. By Bleicher et al., page 4497. Bacterial surface-ripened cheeses ("red-smear cheeses") constitute an important proportion of artisan-produced, highquality foods in Europe. Some of the stable but mostly undefined ripening floras are able to inhibit the human pathogen Listeria monocytogenes, which finds suitable growth conditions on those cheeses. A variety of antibacterial compounds produced by individual bacterial strains have been identified in the past. This study investigated the production of antilisterial substances by a complex ripening consortium after a short-term liquid culture. The data indicate the involvement of yet unknown, small, heat-stable molecules, which are different from bacteriocins.

Bactericidal activity of lauric arginate in milk and Queso Fresco cheese against Listeria monocytogenes cold growth. By Soni et al., page 4518. Queso Fresco cheese (QFC) is a high-moisture, Mexican-style soft cheese that has been classified within FDA cluster 1 of ready-to-eat food products, which indicates that it is one of the highest risk foods with respect to Listeria monocytogenes contamination. Control measures for QFC during its cold storage are inadequate because this soft cheese has frequently been associated with class 1 recalls due to L. monocytogenes contamination. In this study, GRAS (generally regarded as safe) lauric arginate (LAE) was evaluated for its antimicrobial effectiveness against $L$. monocytogenes cold growth in milk and QFC. Our findings revealed that the LAE is able to exert listericidal activity towards L. monocytogenes cold growth at $4^{\circ} \mathrm{C}$ during the initial application stage without affecting the sensory quality of QFC.

Bifidobacterium bifidum BF-1 suppresses Helicobacter pylori-induced genes in human epithelial cells. By Shirasawa et al., page 4526. We have shown that the probiotic strain Bifidobacterium bifidum BF-1 suppresses the expression of inflammatory genes induced by Helicobacter pylori. Probiotic treatment may replace the antibiotic treatment of $H$. pylori in the future. 
Influence of milk pretreatment on production of free fatty acids and volatile compounds in hard cheeses: Heat treatment and mechanical agitation. By Vélez et al., page 4545. Reggianito is an Argentinean traditional hard-cooked cheese and it represents an important share of cheese exports as well as the domestic market. Innovations in cheese making process have been undertaken to improve flavor and accelerate ripening. This work was aimed at assessing the influence of different pretreatments of cheese-milk on fat hydrolysis and volatile compound production in hard cheeses. Results suggest that heat treatment had a greater effect on cheese lipolysis and volatile compound production than partial destabilization of the fat emulsion produced by the agitation method applied.

Rheological properties and microstructure of Cheddar cheese made with different fat contents. By Rogers et al., page 4565. Fat reduction in cheese is desired but can be problematic because of the diminished textural quality. We investigated what happens when the general composition of the nonfat phase is held constant, and the fat and nonfat phases are adjusted. Increased fat content was associated with increased size of fat globules and a less spherical shape. The overall firmness of cheese depended on temperature, and differences due to fat content diminished when cheese was evaluated at room temperature. This suggests that diminished textural quality in low-fat cheese is due to changes in the breakdown pattern during chewing, as altered by fat disrupting the cheese network.

Short communication: Relationships between $\alpha$-lactalbumin and quality traits in bulk milk. By Wickström et al., page 457\%. Bulk milk quality is of major importance for all dairy processors, because it has an effect on yield and quality of dairy products. In the present study, the whey protein $\alpha$-lactalbumin was evaluated as an alternative to somatic cell count as a marker for bulk milk quality in relation to mastitis. This research can help improving existing quality systems for raw milk. Results showed, however, that $\alpha$-lactalbumin was not a sensitive marker for bulk milk quality compared with the conventional marker, somatic cell count.

Use of an internal teat sealant in combination with cloxacillin dry cow therapy for the prevention of clinical and subclinical mastitis in seasonal calving dairy cows. By Runciman et al., page 4582. A combination dry cow therapy using $600 \mathrm{mg}$ of cloxacillin with teat seal was compared with the use of $600 \mathrm{mg}$ of cloxacillin alone in 2,013 dairy cows. The incidence and cause of clinical mastitis was recorded along with herd test records to evaluate somatic cell counts. The cows that received the combination treat- ment had a significantly lower incidence of clinical and subclinical mastitis in early lactation. The difference between treatment groups was largest in cows with a prior history of mastitis. The use of combination dry cow therapy is likely to be of economic benefit on many dairy farms.

Activin-related proteins in bovine mammary gland: Localization and differential expression during gestational development and differentiation. By Bloise et al., page 4592. Activins are members of the transforming growth factor $\beta$ (TGF- $\beta$ ) superfamily. In animal models, TGF- $\beta$ superfamily members regulate mammary development and differentiation, suppression of lactation, and postlactation involution. In the present paper we evaluated gene and protein expression of activin-related proteins in the mammary gland of nonpregnant and pregnant heifers. The present study demonstrated that activin-related proteins display different patterns of expression during bovine gestational mammary gland development and differentiation. The modulation of the expression pattern during gestation suggests that activin-related proteins may play a key role in regulating bovine mammary branching morphogenesis and epithelial differentiation.

The effect of selenium supplementation before calving on early-lactation udder health in pastured dairy heifers. By Ceballos et al., page 4602. Suboptimal selenium (Se) intake has been associated with lowered resistance to mastitis. Either long-acting injectable barium selenate or daily oral Se yeast was administered to pastured dairy heifers before calving. Supplementation, regardless of Se source, increased glutathione peroxidase activity, tended to lower prevalence of intramammary infection, and reduced somatic cell count shortly after calving, but had no effect on the risk of new intramammary infections or on somatic cell count in the first month of lactation. Precalving Se supplementation appears beneficial for improving udder health shortly after calving in heifers grazing low-Se pastures.

Effect of cytochrome $\mathrm{P} 450$ and aldo-keto reductase inhibitors on progesterone inactivation in primary bovine hepatic cell cultures. By Lemley and Wilson, page 4613. Progesterone is required for maintenance of pregnancy. In the lactating dairy cow, the rate of progesterone inactivation has been associated with liver blood flow and cytochrome P450 activity. Few studies have addressed the contributions of hepatic cytochrome P450 enzymes to total progesterone inactivation. By studying specific enzyme inhibitors in bovine hepatic cell cultures, we determined that cytochrome P450 enzymes and aldo-keto reductase enzymes contributed approximately 55 and $40 \%$ of the total progesterone inactivation, respectively. Future work 
focusing on decreasing the activity of these enzymes in vivo could lead to an increase in the bioavailability of progesterone.

Molecular characterization of Prototheca strains isolated from Italian dairy herds. By Ricchi et al., page 4625. One hundred sixty-one Prototheca spp. strains isolated from composite milk and barn-surrounding environmental samples (bedding, feces, drinking/washing water, swabs) of 24 Italian dairy herds were characterized by genotype-specific PCR analysis. The role of Prototheca zopfii genotype 2 as a mastitis pathogen was confirmed, whereas Prototheca blaschkeae was only sporadically isolated from milk. Likewise, $P$. zopfii (genotypes 1 and 2) and P. blaschkeae were isolated from the environment. These data contradict the previous hypothesis that $P$. zopfii genotype 2 was the exclusive causative agent of protothecal mastitis and, on the contrary, confirm that such pathology could be caused by $P$. blaschkeae in a few incidences.

Effect of inseminating cows in estrus following a presynchronization protocol on reproductive and lactation performances. By Chebel and Santos, page 4632. Voluntary waiting periods (VWP) of cows were predetermined to be either 49 (short VWP, SVWP) or 72 (long VWP, LVWP) d postpartum. Cows in the SVWP group were inseminated in estrus after presynchronization and submitted to a timed artificial insemination (AI) protocol if not observed in estrus; LVWP cows were all submitted to a timed AI protocol. The interval from parturition to first AI was 9.5 d shorter for SVWP cows. Pregnancies per AI after first postpartum AI and the rate at which cows became pregnant were not different. Cows that resumed ovarian cycles by $49 \mathrm{~d}$ in milk (DIM) became pregnant at a faster rate than those that resumed cyclicity by 62 DIM and those anovular by 62 DIM. Length of VWP did not affect milk yield, but cows that conceived later in lactation had the greatest milk yield. Inseminating cows in estrus following a presynchronization protocol did not affect reproductive or lactational performances.

Output of selenium in milk, urine, and feces is proportional to selenium intake in dairy cows fed a total mixed ration supplemented with selenium yeast. By Walker et al., page 4644 . The amounts of selenium yeast fed to cows to produce selenium-enriched milk and dairy products may be 20 to 40 times higher than that used to prevent selenium deficiency. Nutritional strategies for the production of selenium-enhanced milk need to be developed to provide target concentrations of milk selenium. Not all dietary selenium is secreted in milk, however, with considerable quantities being excreted in urine and feces. The period for which the diet of cows is supplemented with large amounts of selenium yeast will need to be limited to avoid accumulation of selenium on dairy farms.
Activation of liver $\mathrm{X}$ receptor (LXR) enhances de novo fatty acid synthesis in bovine mammary epithelial cells. By McFadden and Corl, page 4651. Activation of liver X receptor (LXR) regulates lipid and cholesterol synthesis in rodent species. In this study, activation of LXR enhanced de novo fatty acid synthesis in bovine mammary epithelial cells. Development of new technologies that give producers the ability to modify milk composition to meet consumer demand is needed. Identifying LXR as a regulator of lipid synthesis may result in the development of new technologies and management strategies to control milk fat production in lactating dairy cows.

The Garfagnina goat: A zootechnical overview of a local dairy population. By Martini et al., page 4659. This is the first report of a zootechnical overview of the Italian goat population "Garfagnina", which is registered on the Tuscan regional repertory of genetic resources at risk of extinction. Data varied on the size of the goats, zoometric data, breeding system, milk quality, and genetic polymorphisms.

Associations between cow hygiene, hock injuries, and freestall usage on US dairy farms. By Lombard et al., page 4668. This study evaluated management factors associated with hygiene, hock injuries, and stall use in freestall dairies across the United States. Hygiene was better on farms that did not dock tails. Stall design and maintenance were important for prevention of hock lesions and stall use. Freestalls with rubber mats or mattresses were associated with severe hock injuries and increased standing in stalls; the use of sand and frequent bedding was associated with increased lying and fewer hock injuries. Farms in the western United States had cleaner cows and fewer hock injuries compared with farms in the eastern United States.

Use of individual cow milk recording data at the start of lactation to predict the calving to conception interval. By Madouasse et al., page $467 \%$. Milk quantity and composition in the first 2 mo of lactation were used to predict the calving to conception interval in a large sample of herds from England and Wales. Longer intervals between calving and conception were associated with higher milk yield, lower percentage of protein, and higher somatic cell count on the second milk recording as well as lower percentage of lactose, higher somatic cell count, and higher percentage of butterfat on the first milk recording of lactation. Yield protein and lactose were better predictors of the calving to conception interval than the commonly used fat to protein ratio.

Blood profiles in dairy cows with displaced abomasum. By Stengärde et al., page 4691. Blood profiles and a metabolic index for insulin sensitivity were stud- 
ied in dairy cows diagnosed with a displaced abomasum within the first 56 days of lactation. Major differences in metabolism, indicating negative energy balance, liver cell damage, and an inflammatory response, were found in cows with displaced abomasum compared with healthy herd mates sampled at the same time relative to calving. The results contribute to an understanding of the metabolic changes in cows with displaced abomasum.

Diagnosis and treatment of subclinical mastitis in early lactation in dairy goats. By McDougall et al., page 4710. Dairy goats with one or both glands with a positive California Mastitis Test were selected for milk culture. Goats with one or both glands with intramammary infection were assigned to be treated with 3 intramammary infusions of ampicillin and cloxacillin or left as untreated controls. Treatment resulted in a higher bacteriological cure rate and a lower gland-level somatic cell count compared with no treatment. However, treatment had no effect on subsequent composite somatic cell count, milk yield, or length of lactation and was therefore not cost effective.

Localization of Mycobacterium avium ssp. paratuberculosis in artificially inoculated milk and colostrum by fractionation. By Van Brandt et al., page 4722. Mycobacterium avium ssp. paratuberculosis (MAP) causes Johne's disease in cattle and might be linked to Crohn's disease in humans. Colostrum and milk of infected cows are possible transmission routes to calves and humans. This study evaluates the extent to which MAP associates with the cream, curd, and whey fractions of milk and colostrum after fractionation. We found that MAP mainly associates with cream and curd, indicating its possible concentration in specific dairy products. It associates much less with whey, which contains vital immunoglobulins; this could lead to an immunoglobulin-preserving elimination strategy for MAP that the farmer can apply independently to help decrease dissemination of MAP infection.

Short communication: Analysis of milk yield and composition for dairy heifers limit-fed lower forage diets during the rearing period. By Zanton and Heinrichs, page 4730. A limit feeding heifer nutrition management system may improve the efficiency of raising dairy heifers. A mixed-effects meta-analysis was conducted to evaluate the effect of limit feeding energydense rations on first-lactation milk production. Average daily gain did not differ between limit-fed heifers and heifers fed the control diets. First-lactation milk and milk component yield did not differ between cows fed the treatment and control diets during rearing. It is concluded that first-lactation performance is not different between cows that were fed the control treatment from those that were reared by limit feeding.
Inclusion of psyllium in milk replacer for neonatal calves. 2. Effects on volatile fatty acid concentrations, microbial populations, and gastrointestinal tract size. By Cannon et al., page 4744. Psyllium, a viscous fermentable fiber, increases gut volatile fatty acid (VFA) concentrations and gastrointestinal mass. Newborn calves were fed milk replacer without or with $1.1 \%$ psyllium supplementation through $28 \mathrm{~d}$ of age. Psyllium increased colonic VFA concentrations, gastrointestinal tract mass, and density of small intestinal tissue. Psyllium did not affect populations of beneficial bacteria (bifidobacteria and lactobacilli) in the intestines, but increased their populations in the reticulo-rumen. Greater mass and density of the small intestine could increase digestive capacity and resistance to pathogens in milk-fed calves. Increased bifidobacteria and lactobacilli in the reticulorumen might increase starter intake and speed rumen epithelial development.

Effect of induction of subacute ruminal acidosis on milk fat profile and rumen parameters. $B y$ Colman et al., page 4759. This paper describes modifications in milk fatty acid proportions, particularly odd- and branched-chain fatty acids and rumen biohydrogenation intermediates, associated with rumen parameters during a 6 -wk subacute ruminal acidosis induction protocol. A $\mathrm{pH}$ decrease occurred after the first concentrate switch but rumen parameters returned to the original values and remained stable during the first $4 \mathrm{wk}$, whereas in wk 5 and 6 rumen $\mathrm{pH}$ values were indicative of increasing acidotic conditions. A discriminant analysis indicated the main fatty acids discriminating between the control and acidotic samples as iso C13:0, iso C16:0, and C18:2 cis-9,trans-11.

Effects of supplementing dairy cows with chromium propionate on milk and tissue chromium concentrations. By Lloyd et al., page 4774. Chromium $(\mathrm{Cr})$ is a trace mineral that functions by enhancing the action of insulin. Previous studies have indicated that Cr supplementation can increase milk production in dairy cows. This study evaluated the safety of supplemental $\mathrm{Cr}$ propionate in regard to potential $\mathrm{Cr}$ residues in milk and meat. Supplementation of $\mathrm{Cr}$ propionate at a level $(2.0 \mathrm{mg}$ of $\mathrm{Cr} / \mathrm{kg}$ of dry matter) that exceeded the concentration $(0.5 \mathrm{mg} \mathrm{Cr} / \mathrm{kg}$ of dry matter) permitted by the Food and Drug Administration by 4 -fold did not affect $\mathrm{Cr}$ concentrations in milk, muscle, or fat, the major bovine products consumed by humans.

Abomasal or ruminal administration of flax oil and hulls on milk production, digestibility, and milk fatty acid profile of dairy cows. By Kazama et al., page 4781. Administration of flax oil and flax hulls in the rumen compared withadministration in the 
abomasum decreased the proportion of linolenic acid in milk fat but the proportion was two times higher than values reported in previous studies. All ratios of n-6:n-3 fatty acids in milk fat were lower than the 4:1 ratio ideal for human health. The presence of both flax oil and flax hulls in the rumen decreased yield of $4 \%$ fat-corrected milk and digestibility but improved milk fatty acid profile, which may contribute to improved human health.

Effect of feed sorting on chewing behavior, production, and rumen fermentation in lactating dairy cows. By Maulfair et al., page 4791. A study was conducted to evaluate the sorting behavior of lactating dairy cows when fed rations varying in particle size. The influence of sorting on chewing behavior, milk yield, milk components, and rumen fermentation was analyzed. The rations were sorted extensively and to different degrees when sorting was determined by analyzing refusals. However, the cows actually consumed similar amounts of neutral detergent fiber and starch within $24 \mathrm{~h}$ after feeding. Therefore, when evaluating ration sorting in dairy cows, it is important to determine actual consumption of ration components rather than concentration of components in refusals.

Effect of fish oil and sunflower oil on rumen fermentation characteristics and fatty acid composition of digesta in ewes fed a high concentrate diet. By Toral et al., page 4804. Sunflower oil and fish oil (SFO) in the diet are known to modify milk fat composition due to changes in ruminal biohydrogenation, but the intermediates formed are not well characterized. Samples of rumen digesta were collected on d 0, 3, and 10 from ewes fed a high concentrate diet containing SFO to examine the temporal changes in fermentation characteristics and fatty acid composition. Oils had no detrimental effect on ruminal digestion, but caused time-dependent decreases in the relative abundance of 18:0 and 18:2 n- 6 and increased the accumulation of trans 16:1, trans 18:1, trans 18:2, 20, and 22 carbon biohydrogenation intermediates.

Effects of stage of lactation and dietary concentrate level on energy utilization by Alpine dairy goats. By Tovar-Luna et al., page 4818. A complete understanding of energy requirements is necessary for optimal performance of lactating dairy goats. Limitations may exist in approaches to study energy use by lactating goats through assessment of maintenance needs of nonlactating animals. Efficiency of use of dietary energy for lactation differed among early, mid, and late stages of lactation, but the combined efficiency for maintenance and lactation was similar among periods. Efficiencies of dietary energy use for lactation and maintenance plus lactation were greater for a high than for a low concentrate diet. Predicted milk yield based on energy requirements of the National Research Council was reasonably accurate.

Effects of stage of lactation and level of feed intake on energy utilization by Alpine dairy goats. By Tovar-Luna et al., page 4829. A complete understanding of energy requirements is necessary for optimal performance of lactating dairy goats. The energy requirement for maintenance relative to metabolic size in late lactation was less than in early and mid lactation, but with a similar requirement in megajoules per day this difference may have been partially attributable to tissue energy gain throughout lactation. Method of determination can influence the maintenance energy requirement and efficiencies of energy use for maintenance and lactation. Level of feed intake, such as restricted intake after ad libitum consumption, can have a substantial effect on estimates of energy utilization by lactating dairy goats.

A single mild episode of subacute ruminal acidosis does not affect ruminal barrier function in the short term. By Penner et al., page 4838. Subacute ruminal acidosis is a common digestive disorder in beef and dairy cattle. This study aimed to understand whether the barrier function of the ruminal lining (the epithelium) is changed immediately after a mild episode of subacute ruminal acidosis (nadir $\mathrm{pH}$ 5.48). Based on a combined in vivo and in vitro approach, we conclude that such changes are not evident. However, barrier function was persistently compromised after a rapid and more severe ruminal acidification in vitro (pH 5.2).

Short communication: In vitro ruminal fermentability of a modified corn cultivar expressing a thermotolerant $\boldsymbol{\alpha}$-amylase. By $\mathrm{Hu}$ et al., page 4846. The enzyme activity and ruminal fermentability of a modified corn grain expressing a thermotolerant $\alpha$-amylase were evaluated. Amylase activity was relatively high (comparable to the isogenic negative control corn) when ground corn containing $\alpha$-amylase was incubated in water at $40^{\circ} \mathrm{C}$ but was markedly higher when the incubation temperature was $65^{\circ} \mathrm{C}$ (greater than 10 times that of the isogenic negative control corn). Incubating ground corn that contained the $\alpha$-amylase in ruminal fluid at $40^{\circ} \mathrm{C}$ did not alter the amount of volatile fatty acid production or starch disappearance during 6 -h in vitro ruminal fermentations.

Short communication: Effect of grazing on concentrations of total sialic acid and hexose in bovine milk. By Asakuma et al., page 4850. Sialic acid concentration in bovine milk varies depending on lactation stage and season. However, it remains unclear whether dietary factors affect the total sialic acid concentration in milk. The purpose of the present 
study was to investigate the effect of grazing on the concentrations of total sialic acid and hexose in bovine milk. Total sialic acid concentration in the milk of each grazing cow significantly increased compared with that at $0 \mathrm{~d}$ of the experimental period. These results indicate that grazing management could have increased the concentration of sialoglycoconjugates in milk.

Technical note: Precision and accuracy of in vitro digestion of neutral detergent fiber and predicted net energy of lactation content of fibrous feeds. By Spanghero et al., page 4855. A rotating jar in vitro system is available for routine measurement of the fiber digestibility of feeds for ruminants. Feed samples inserted in filter bags are immersed in diluted rumen fluid and maintained in anaerobic conditions. The method has high accuracy compared with in situ data obtained from the same samples and bags incubated in the rumen of cows, but it is still marginally precise, and methodological improvements are required to reduce variability of measurements.

Identification of single nucleotide polymorphisms in the bovine interleukin-12 and interleukin-23 receptor genes and their associations with health and production traits in Holstein cows. By Skelding et al., page 4860. Identification of variants within genes that are associated with health and production traits could contribute to achieving genetic gain over a shorter interval of time through selective breeding. Interleukin-12 (IL-12) and interleukin-23 (IL-23) receptors are suitable candidate genes for studying disease resistance in dairy cattle because they play a key role in modulating inflammation and the host immune response. In this study, 2 SNP in IL12R-1, 5 SNP in IL-12R-2, and 3 SNP in IL-23R were identified in a Holstein bull population, and SNP in the IL-12R-2 gene were associated with variability in milk and protein yield.

The effect of the number of observations used for Fourier transform infrared model calibration for bovine milk fat composition on the estimated genetic parameters of the predicted data. By Rutten et al., page 4872. The effect of the number of calibration samples on the estimated genetic parameters was established. A strong impact on genetic correlations between predicted and observed fatty acids was found. The use of $n=1,000$ calibration samples resulted in estimated genetic correlations varying within an acceptable range. Replacing expensive observations determined by gas chromatography by inexpensive predictions based on infrared spectra reduced the potential genetic gain to $98,86,96$, and $99 \%$, respectively, for the human health-related fatty acids C14:0, C16:0, C18 unsaturated, and the ratio of saturated to unsaturated fatty acids in dairy breeding schemes. Infrared spectra provide an excellent means to the dairy industry to genetically alter milk fat composition.

Claw health index for Dutch dairy cattle based on claw trimming and conformation data. By van der Linde et al., page 4883. Routinely recorded claw health data were used to develop a genetic selection index for claw health. Claw health data comprised records on 40,536 dairy cows trimmed by claw trimmers and observed claw disorders for 7 traits. Estimated heritabilities for claw health traits ranged from 0.01 to 0.13 . Feet and leg conformation scores were used as predictive traits. The breeding goal for claw health was to reduce costs due to claw disorders. An index for claw health, which included claw health traits and feet and leg conformation traits, had a reliability of $59 \%$ for an average progeny-tested bull in the Netherlands.

Genetic parameters of feed intake, production, body weight, body condition score, and selected type traits of Holstein cows in commercial tiestall barns. By Vallimont et al., page 4892. Genetic analysis of feed intake has historically involved the use of records from research herds. In this study, feed intake was collected on 11 commercial tie-stall dairy farms and heritable variation was found for this trait. Genetic correlations of feed intake with milk yield traits, body weight, and stature were positive and moderate to high. The assessment of genetic parameters of feed intake using measures of this trait collected from commercial tie-stall herds is possible.

Assessment of inbreeding depression in a Guzerat dairy herd: Effects of individual increase in inbreeding coefficients on production and reproduction. By Panetto et al., page 4902. Influences of inbreeding on daily milk yields, age at first calving and calving intervals were determined on a highly inbred zebu dairy herd of the Guzerat breed. Models were compared that fit traditional inbreeding coefficients with models that fit individual increase in inbreeding coefficients. Individual increase in inbreeding has been proposed as an alternative measure of inbreeding to account for the differences among an animal's number of known generations of ancestors.

A genome scan for quantitative trait loci affecting milk somatic cell score in Israeli and Italian Holstein cows by means of selective DNA pooling with single- and multiple-marker mapping. By Tal-Stein et al., page 4913. Udder infection (mastitis) is an important dairy cattle disease affecting animal welfare and milk yield, quality, and safety. The infected udder attracts large numbers of somatic cells (leucocytes) to combat the pathogen. Consequently, milk somatic cell score (SCS) serves as a convenient measure of mastitis. We mapped chromosomal regions 
(QTL) affecting genetic variation in SCS in Israel and Italian Holstein cattle. The number of QTL and their effects explained virtually all genetic variation in this trait. These results will provide a basis for anchoring genetic variation in SCS to specific genes, enabling highly efficient gene-assisted selection for resistance.

Understanding the milk-to-feed price ratio as a proxy for dairy farm profitability. By Wolf, page 4942. The milk-to-feed ratio is examined as a proxy for dairy farm profitability. Recent volatility in feed prices has diminished the utility of this measure. Income over feed costs is presented as an alternative measure that more closely reflects profitability.

Effects of running time of a cattle-cooling system on core body temperature of cows on dairy farms in an arid environment. By Ortiz et al., page 4949. To evaluate the hypothesis that the Korral Kool cattle cooling system should be set to work on the basis of the thermoneutral conditions of dairy cattle, the effects of operating time were evaluated on core body temperature of lactating dairy cows. The Korral Kool system should be operated continuously to decrease heat stress in multiparous cows, but operating time could be reduced from 24 to $21 \mathrm{~h}$ for primiparous cows; this reduction needs to be done carefully.

A comparison of the effects of 2 cattle-cooling systems on dairy cows in a desert environment. By Ortiz et al., page 4955. This study analyzed the differences between 2 sizes of Korral Kool systems and 2 running times on core body temperature of multiparous lactating cows housed in an arid environment. Korral Kool systems should operate continuously in desert environments to decrease the negative effects of heat stress regardless of the size of the system used. At certain times of day, the 5-hp fan system performed better in terms of cooling and resulted in lower mean ambient temperatures than the 3 -hp fan system. The larger system allowed for greater spacing between cooling system units.
"Global" and "local" predictions of dairy diet nutritional quality using near infrared reflectance spectroscopy. By Tran et al., page 4961. Dairy diets were predicted for dry matter intake, quality, and digestibility using fecal near infrared reflectance spectroscopy (NIRS). Fecal NIRS accurately predicts net energy for lactation, crude protein, neutral detergent fiber, acid detergent fiber contents, and the digestibility of these components in the diets. The classic calibration technique (global) that computes and develops a global equation for all unknown, unanalyzed samples was compared with an innovative calibration technique (local) that computes a specific equation for each unknown sample. The predictive accuracy was greatly improved with the local technique, especially on averaged animal group data. The improvement achieved with the new technique is potentially useful for dairy diet evaluation.

Effect of pregrazing herbage mass on methane production, dry matter intake, and milk production of grazing dairy cows during the mid season period. By Wims et al., page 4976. We investigated the effect of pregrazing herbage mass on methane production and animal performance of grazing dairy cows in the mid-season grazing period (June-July). Two levels of herbage mass were offered, with target levels of 1,000 and $2200 \mathrm{~kg}$ of dry matter/ha. This study reported no effect of herbage mass on animal performance. The study also found that implementing a high standard of grazing management, such as by maintaining low pregrazing herbage mass, will reduce methane emissions from grazing dairy cows.

Associations of breed and feeding management with milk production curves at the herd level using a random regression test-day model. By Caccamo et al., page 4986. Milk and milk components in dairy cattle are influenced by many factors, including herd management. This study explained variability in random herd curves in terms of breed, feeding system, and nutrition parameters. 